\title{
El costeo ABC como estrategia de gestión empresarial en el sector de servicios. Caso: Salón los Maderos
}

$A B C$ costing as a business management strategy in the service sector. Case: Salón los Maderos.

Jacqueline Alexandra Guerrero Romero. ${ }^{1}$, Cecilia Ivonne Narváez Zurita. ${ }^{2}$, Jorge Edwin Ormaza Andrade. ${ }^{3}$ \& Juan Carlos Erazo Álvarez. ${ }^{4}$

\section{DOI: https://doi.org/10.33262/visionariodigital.v3i2.1.553}

\begin{abstract}
.
Currently, companies face difficulties in maintaining a market that is increasingly competitive and globalized, being forced to undertake reengineering and restructuring activities to reinvent themselves and adapt to the current problems. The difficult economic situation facing the various countries worldwide, including Ecuador, requires management to seek effective management tools that allow it to cope with the economic crisis and generate useful strategies of competition. In the present work a costing by activities for service companies is proposed, having as an analysis unit the restaurant "Salón los Maderos". With the study of the theoretical foundations of cost management based on ABC / $\mathrm{ABM}$ activities and their influence on financial decision making in service companies,
\end{abstract}

\footnotetext{
${ }^{1}$ Universidad Católica de Cuenca, Posgradista Maestría en Contabilidad y Auditoría, Cuenca, Ecuador, jaguerreror916@psg.ucacue.edu.ec

${ }^{2}$ Universidad Católica de Cuenca, Subdirección de Posgrado Unidad académica de administración, Cuenca, Ecuador, inarvaez@ucacue.edu.ec

${ }^{3}$ Universidad Católica de Cuenca, Subdirección de Posgrado Unidad académica de administración, Cuenca, Ecuador, jormaza@ucacue.edu.ec

${ }^{4}$ Universidad Católica de Cuenca Subdirección de Posgrado, Cuenca, Ecuador, jcerazo@ucacue.edu.ec
} 
the aim is to determine the cost of the products and / or services offered, as well as the profitability of them; for this it is essential to know not only the inputs and resources required by the product and / or service, but also the areas related to them and the activities that involve them; In this way, make decisions correctly. In the restaurant "Salón los Maderos" of the city of Cuenca there are difficulties in terms of cost management, due to the resistance to formalize the processes, using as a single tool "experience" in addition to costs and expenses managed empirically.

Keywords: $\mathrm{ABC}$ costing, strategic, tactical, operational, decision making, processes.

\section{Resumen}

Actualmente las empresas afrontan dificultades para mantenerse en un mercado que cada día es más competitivo y globalizado, viéndose obligadas a realizar actividades de reingeniería y reestructuración para reinventarse a sí mismas y poder adaptarse a la problemática actual. La difícil situación económica que atraviesan los diversos países a nivel mundial, incluido el Ecuador, obliga a la gerencia a buscar herramientas efectivas de gestión que le permitan sobrellevar la crisis económica y poder generar estrategias útiles de competencia.

En el presente trabajo se planteó un costeo por actividades para empresas de servicios, teniendo como unidad de análisis el restaurante "Salón los Maderos". Con el estudio de los fundamentos teóricos de la gestión de costos basados en actividades ABC/ABM y su influencia en la toma de decisiones financieras en las empresas de servicios, se determinó el costo de los productos y/o servicios que ofrece; para ello, fue necesario conocer no sólo los insumos y los recursos que requieren los productos y/o servicios, sino también las áreas relacionadas con ellos y las actividades que los involucra.

En el restaurante "Salón los Maderos" de la ciudad de Cuenca existen dificultades en cuanto a la gestión de costos, debido a la resistencia a formalizar los procesos, usando como 
única herramienta "la experiencia" además de costos y gastos manejados de forma empírica.

Palabras clave: Costeo ABC, estratégico, táctico, operativo, toma de decisiones, procesos.

\section{Introducción.}

La contabilidad de costos responde a la necesidad que tiene la alta gerencia de obtener información específica y no general, además de proveer información para la contabilidad financiera, herramienta que permite verificar o visualizar de qué manera las decisiones de la administración afectaron a la utilización de los recursos de la empresa (Cuervo, Osorio y Duque, 2013). La contabilidad de costos es una herramienta indispensable para la contabilidad financiera, pues a través de esta, se puede cuantificar los elementos de los costos de la materia prima, mano de obra y los gastos generales de producción; permitiendo de esta forma, la toma de decisiones acertadas por parte de los empresarios, pues lo harían en base a los costos totales y unitarios de fabricación de los productos (Altajona, 2009).

La importancia de la contabilidad de costos difiere también dependiendo del tipo de empresa que la utilice, por ejemplo, una empresa que fabrica productos de aseo personal usará la contabilidad de costos como herramienta principal, no siendo así en una empresa que distribuye estos productos fabricados a los minoristas y consumidores finales. Por otro lado, las empresas de servicios tienen inventarios muy pequeños para las cuales la contabilidad de costos sería muy básica (Blocher, Stout, Cokins y Chen, 2008).

La contabilidad de costos utiliza métodos y procedimientos para registrar, resumir e interpretar las operaciones relacionadas con los costos que se necesitan para elaborar un artículo, prestar o vender un servicio de las actividades inherentes a la producción; manifiestan, que es una herramienta financiera de gran utilidad para la alta gerencia de las empresas productoras de bienes o servicios (Gómez y Zapata, 1998). 
Los costos se clasifican de acuerdo a su comportamiento y por su naturaleza o actividad operacional: por órdenes de producción y por procesos; por departamentos o secciones: costos de administración y costos de ventas; por el método del cálculo: costos reales, costos predeterminados y costos estándar; por el volumen de producción: costos fijos, costos variables y costos semivariables; por la identificación del producto: costos directos y costos indirectos (Sarmiento, 2005).

\section{Costos ABC/ABM}

Autores como: Adame (2000), Cuevas (2001), Bendersky (2002), Pérez (2009), Cuervo, Duque y Osorio (2013), han escrito acerca de la gestión de costos por actividades, la mayoría de ellos concuerdan en que ésta nace luego de que diferentes administrativos notan que, no es útil el solo hecho de sumar los costos más la utilidad para saber el precio de venta, pues es necesario cambiar esa ecuación tradicional, por un enfoque con mayor profundidad que ayude a la correcta identificación del uso de los recursos.

El costeo basado en actividades ( $\mathrm{ABC}$ ) y la administración basada en actividades (ABM), aparecen como una respuesta a la necesidad de responder a una época de empresas automatizadas, donde la tecnología cambia totalmente el sistema tradicional de costeo. El tener la información del costo de horas hombre, no era suficiente para la toma de decisiones financieras, es así, que surge el costeo basado en actividades como una herramienta que genera información confiable para que los directivos puedan competir al poder manejar sus costos basados en actividades que usan recursos los cuales se reflejan en el precio del producto o servicio (Padilla, 2008).

La gestión de costos tiene grandes ventajas ya que no se limita solo a establecer los costos o a mejorarlos, pues luego de un sistema de costeo bien diseñado como lo es el $\mathrm{ABC}$ y con la información que este nos provee, los administradores pueden mejorar los beneficios que los clientes reciben y al mismo tiempo incrementar las ganancias del ente económico, esto es lo que logran aquellas empresas que han optado por la administración basada en actividades (Blocher, Stout, Cokins y Chen, 2008). 
La gestión basada en actividades (ABM) tiene su base en el volumen de producción por lo que el sistema $\mathrm{ABC}$ es más preciso en la asignación de los costos indirectos de fabricación. No obstante, para que el costeo $\mathrm{ABC}$ llegue a ser una verdadera herramienta gerencial necesita una correcta administración del mismo conocida como gestión ABC (Cuevas, 2001). El sistema de costeo ABC asigna todos los costos corrientes a los productos, sin analizar si la actividad agrega valor o no al producto dentro de la cadena de valor. Una visión gerencial trata de reducir o eliminar estos costos que no representan valor agregado (Cuevas, 2001).

De acuerdo a Cooper y Kaplan (1991) la administración basada en actividades tiene dos enfoques marcados como objetivos:

$\checkmark$ El ABM operativo: su objetivo es que las actividades se realicen correcta y eficientemente o de otra forma se dice que es intentar mejorar las actividades que se realizan, debido a esto, es necesario la gestión de actividades, reingeniería de procesos, calidad total y por último evaluar las actividades.

$\checkmark$ El ABM estratégico: el objetivo de este enfoque se basa en seleccionar las actividades que deben desarrollarse, en consecuencia, las acciones de corrección serían el diseño del producto, tipo de productos y clientes, las relaciones con la cartera de clientes, los precios, segmentar de forma correcta el mercado y establecer los canales de distribución.

Pérez (2015) afirma que el ABM está ligado al ABC, en razón que este último se encarga de calcular los costos de los servicios o productos, mientras que, el primero usa como base los resultados de los cálculos para la estimación de costos y es por esta razón, que cuando se habla de los dos como un conjunto se usa el término $\mathrm{ABC} / \mathrm{M}$. En este contexto, es necesario precisar ciertas definiciones de elementos que provee este último:

- ABA: Activity based accounting, que supone un sistema de organización contable basada en las actividades.

- ABB: Activity based budgeting, que es un sistema de presupuesto y de provision basado en las actividades. 


\section{Bases y componentes teóricos del costeo $\mathrm{ABC}$}

El costeo basado en actividades mide el costo del producto terminado y el desempeño de actividades, recursos y objetos del costo. Los recursos se asignan primero a las actividades, después, los costos de las actividades se asignan a los objetos del costo según su uso. Los niveles de costo y guías de asignación se usan en el sistema $\mathrm{ABC}$ para distribuir los costos indirectos de fabricación. El costeo ABC reconoce actividades, costos de las actividades y guías de asignación de estas a diferentes niveles de agregación dentro del medio productivo (Cuevas, 2001).

\section{$\checkmark$ Recursos}

Para Uribe (2011) los recursos son variados pero los principales son tres; nómina, equipo de oficina y los materiales. Sin embargo, desde el punto de vista de la gestión de costos $\mathrm{ABC}$ se pueden distinguir en dos grupos uniformes; los primeros son aquellos que se encuentran en la información que da la contabilidad financiera los cuales el autor lo define como los recursos contables al estar dentro de cualquiera de los estados financieros, y por otra parte, aquellos que se define como los recursos no contables pues no tienen la obligación de cumplir los Principios de Contabilidad Generalmente Aceptados (PCGA). Cuervo, Osorio y Duque (2013) mencionan que los recursos son aquellos elementos o medios que son consumidos por todas las actividades desarrolladas.

\section{$\checkmark$ Actividades}

Para Uribe (2011) quien concuerda con Cuervo, Duque y Osorio (2013) manifiestan que estas son un grupo uniforme de acciones de una o varias personas las cuales consumen los recursos físicos, humanos y tecnológicos en la producción de un bien o en la prestación de un servicio, con el objetivo de presentarlos a usuarios internos o externos. Estas se clasifican de acuerdo a criterios como; la categoría a la que pertenecen, según sus funciones, a la participación en el costo, a su periodicidad, y a su aportación a la generación de valor.

Se detallan las actividades según la participación en el costo, las mismas que se clasifican en actividades primarias y secundarias. Dentro de las primarias se encuentran aquellas que están relacionadas directamente con el bien o servicio, para lo cual se usa como ejemplo las siguientes: selección del proveedor, pedido de insumos, ingreso a bodega, registro en el kárdex, toma de orden, 
secuencia del servicio (control de tiempos). Las actividades secundarias son importantes para poder alcanzar los objetivos de la empresa, estas soportan a las actividades primarias, se puede mencionar las siguientes para ejemplificar las mismas: elaboración y pago de nóminas, preparar informes contables, preparar las declaraciones tributarias, realizar la planeación financiera.

\section{$\checkmark$ Inductores de costos}

Conocidos como drivers, direccionadores, inductores o cost driver. Estos son aquellos vínculos que conectan las actividades con los recursos de manera que se puedan cuantificar la frecuencia y la intensidad de estos, para de esa forma, realizar la respectiva asignación de los de más alto costo a las actividades (Adame, 2000). Por otra parte, Cuervo, Duque y Osorio (2013) los definen como aquellos parámetros o criterios de aplicación que son usados para poder realizar la respectiva asignación de los costos, y la medición razonable del uso de los recursos, áreas de responsabilidad, y de las actividades entre otros.

\section{Objetos de costo}

Para Horngren, Datar y Rajan (2012) es todo aquello para lo cual se necesite medir o cuantificar el costo, podría ser, de uno o varios productos, de un servicio o de alguna cosa en particular. Se puede citar como ejemplo de bjetos de costo de: un producto es una bicicleta, un servicio la asistencia de un call center para atención al cliente, un proyecto para investigar el mercado de una nueva línea de productos, la actividad del mantenimiento de todas las computadoras en el área de contabilidad, y el costo de un departamento de recursos humanos parala empresa, entre otros.

\section{$\checkmark$ Cadena de valor}

Es una sucesión de situaciones donde se agrega a los bienes una ganancia para el comprador, las situaciones importantes de esta sucesión son las siguientes: investigación y desarrollo, diseño del producto y de los procesos, producción, marketing, distribución y servicio al cliente (Horngren, Datar y Rajan (2012) 


\section{Metodología}

El diseño investigativo tuvo una orientación no experimental, ya que no se incidió en las variables de estudio; la presente investigación se desarrolló con un enfoque mixto con el propósito de dar cumplimiento al objetivo planteado; el enfoque cualitativo se justifica en razón que se usaron técnicas y procedimientos de recolección y análisis de información relacionada al marco teórico que sustenta el artículo y cuantitativo ya que se recogieron e interpretaron datos contables y estadísticos propios del giro del negocio, el alcance del documento fue descriptivo - explicativo de tipo transversal y de campo.

Los métodos que se utilizaron fueron el inductivo - deductivo, porque partió del análisis de los conceptos generales existentes de las empresas de servicios para luego ir a lo particular que son los restaurantes de especialidades; además, se estableció una relación entre la gestión de costos por actividades y la toma de decisiones financieras analizando la realidad específica para luego proveer de conceptos generales; de la misma forma se recurrió al método analítico - sintético para entender todos los componentes de la gestión de costos por actividades; los instrumentos para la obtención de información de fuentes primaria fueron la observación directa, entrevista y encuesta, como población de análisis se consideró a todo el personal que labora en la organización; es decir, en términos estadísticos se trata de una población finita.

\section{Tratamiento estadístico y representación gráfica}

A partir de la aplicación de los instrumentos de la investigación se obtuvieron los siguientes resultados:

\section{$\checkmark$ Nivel estratégico}

Los procesos estratégicos, ayudan a la empresa a determinar métodos de diferenciación por ventajas competitivas o comparativas.

El Salón los Maderos, cuenta con una estrategia conocida como orientación vertical hacia atrás, lo que significa que la empresa tiene un criadero propio de cerdos, que es su materia prima, situación que le permite abastecerse en temporadas altas, y cuando éstos no están listos para su consumo, 
dispone de proveedores quiénes cumplen con los requisitos de calidad exigidos por el propietario. El correcto conocimiento de cómo se enlazan los procesos con las actividades específicas ayuda a determinar un costeo adecuado por actividades.

\section{$\checkmark$ Nivel táctico}

En el orgánico funcional de las empresas se encuentran ubicados a nivel táctico todos aquellos encargados de tomar las decisiones de la producción, ventas y administración, entre otras, así como también diseñar las actividades de los colaboradores que se encuentran en el nivel inferior que es el operativo.

En el Salón los Maderos, se observó y comprobó que no existe una planificación en cuanto a las actividades de producción y venta, estas se realizan al momento de recibir los pedidos por parte de los clientes, y los realiza la administración personalmente. Lo que limita a los colaboradores de nivel inferior a estar preparados en temporadas altas de venta.

\section{$\checkmark$ Nivel operativo}

En la parte operativa se maneja el control de la materia prima, los alimentos que se sirven, la forma de manejar los pedidos, la forma en que está organizada la estructura de la cocina, la infraestructura del local y el almacenamiento de los productos. Se debe analizar con mucho cuidado la forma en que un cliente es tratado y cuál fue su experiencia al ser atendido por el personal de la organización.

En la unidad de análisis se detectó que se contrata personal sin tomar en cuenta su experiencia o estudios, así como no se se realiza procesos de inducción y capacitación a los mismos.

No se cuenta con una gestión de calidad, manuales de procedimientos o formas de gestionar la atención recibida por parte de los clientes. En la entrevista realizada al gerente supo manifestar que no consideraba importante estos aspectos debido a que su producto era bueno por el buen sabor y lo fresco de las vegetales y guarniciones con las que servía sus platos.

\section{$\checkmark$ Costos principales}

En una empresa es importante conocer los costos de la materia prima directa, mano de obra directa y los costos indirectos de fabricación del bien o servicio que se comercialice. Dentro de estos 
costos se encuentran las actividades principales del proceso productivo dentro de la empresa, y constituye los servicios específicos que presta esta.

Se constató que el restaurante no cuenta con un sistema adecuado de gestión de costos que permita identificar las actividades principales mucho menos los costos de las mismas, como lo sustenta cuando se comprobó que las únicas herramientas que los administradores usan para la toma de decisiones financieras son los ingresos versus los gastos, esto resulta del manejo empírico de la información, y del uso de la experiencia.

\section{$\checkmark$ Costes auxiliares}

Aquí se encuentran las actividades de apoyo como son las de mercadeo y administrativas. Este es el lugar en donde se generan los costos indirectos, que constituyen un importante porcentaje de la carga final al costo unitario para la determinación de precios, con lo que permite establecer el costo del producto.

El Salón Los Maderos cuenta con los servicios profesionales del área contable como medio para pagar nómina, presentar informes requeridos por los entes de control y realizar las declaraciones de impuests, por lo que se corroboró, que no se utilizan la información contable para la toma de decisiones financieras.

\section{Propuesta.}

El Salón los Maderos de la ciudad de Cuenca es un emprendimiento que ha permanecido con el tiempo, a pesar de no contar con una gestión de costos adecuada, ha logrado superar algunos obstáculos propios del mercado en el que se desenvuelve. Sin embargo, no ha alcanzado el crecimiento que podría tener al contar con un modelo de gestión de costos adecuado.

La propuesta del presente artículo está encaminada a identificar las actividades que se realizan en la empresa objeto de estudio, con el fin de que éstas se ejecuten de forma correcta, lo cual se consigue luego de identificar los procesos para luego establecer los centros de costos y de esta forma establecer si estas se cumplen y, en consecuencia a las consideraciones anteriores, se 
establecerán los costos que sean necesarios para brindar un servicio de calidad a los clientes así como ofrecer herramientas útiles para la toma de decisiones por parte de los directivos.

Figura 1: Propuesta de Costeo ABC Salón Los Maderos

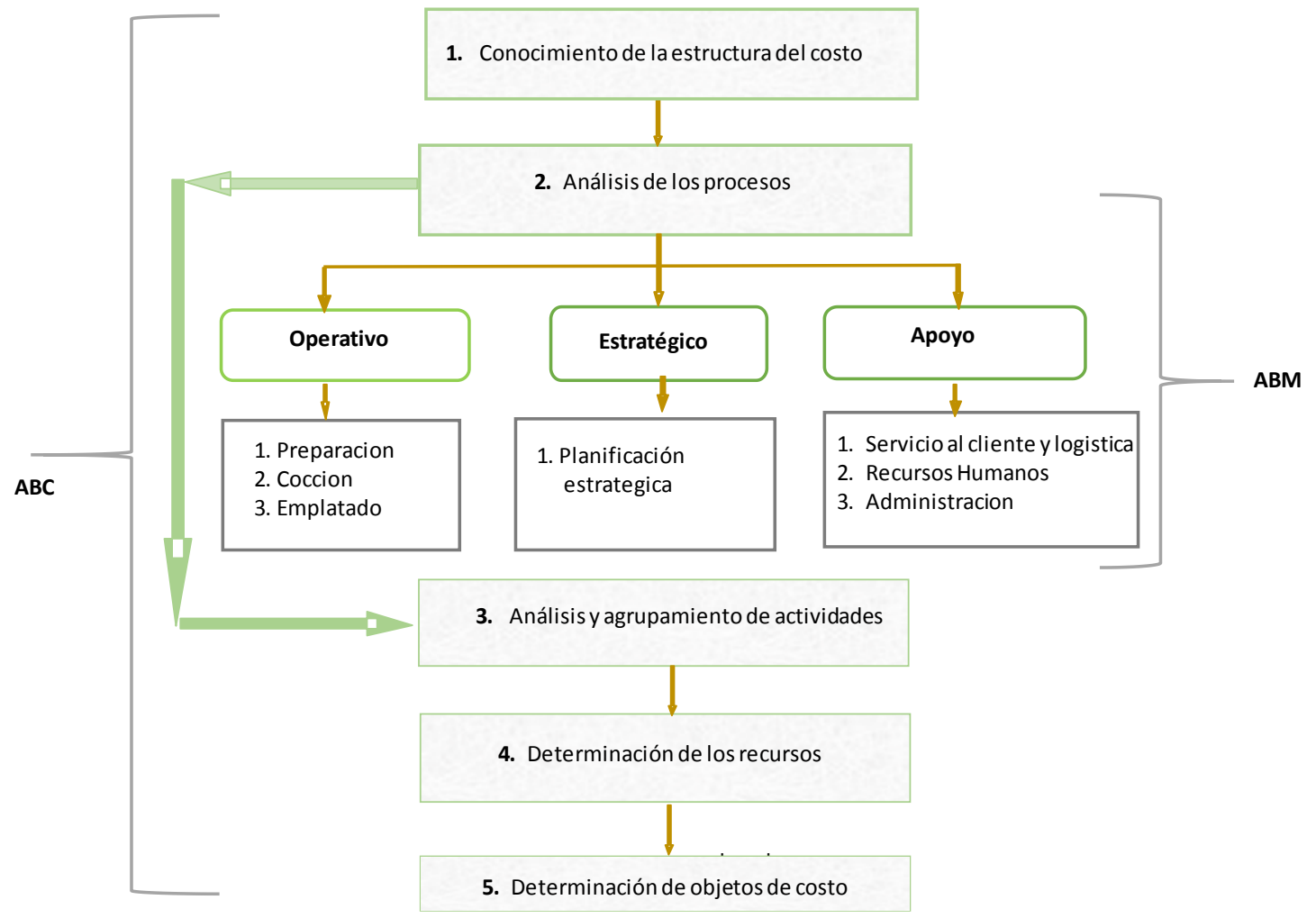

\section{Procesos operativos}

Grupo de actividades que permiten brindar el servicio de comida, los procesos operativos manejan el control de la materia prima, mano de obra directa y los procesos directamente relacionados con la actividad de la empresa.

Preparación, todo plato empieza desde el ingreso de la materia prima a la cocina y su correcta manipulación.

Cocción. Luego de ser preparados los ingredientes, se procede a su cocción por distintos métodos. Emplatado, tan importante como los anteriores procesos, es el emplatado y servicio al cliente. 


\section{Procesos estratégicos}

Una vez que se han establecido los procesos estratégicos del restaurante, y tras haber realizado el conocimiento del negocio es preciso plasmar la razón de ser de éste en una misión y visión.

Misión. - Ser el lugar favorito a nivel nacional siendo promotores del turismo nacional e internacional a través de la gastronomía, conservando la tradición y los secretos culinarios en cada receta, satisfaciendo el paladar de nuestros clientes, ofreciendo alimentos y servicio de alta calidad. Con una alineación excepcional cliente interno - externo, productos, lugar, precio justo y promoción. Continuar promoviendo e impulsando el cultivo y mercado orgánico lo que lleva a brindar una alimentación saludable y amigable con el organismo.

Visión. - Impulsar una alimentación con trazabilidad y sustentabilidad preventiva, donde los clientes nos recuerden por la excelencia en servicio, calidad y sabor en un ambiente de armonía, apoyados en el personal con un alto espíritu de servicio.

\section{Procesos de apoyo}

Servicio al cliente y logística, estas actividades agregan valor a un producto o servicio, la gestión de recursos y su correcta logística reduce costos.

Recursos Humanos, el manejo del personal y su adecuada selección es un punto neurálgico en toda institución.

Administración, toda institución requiere de una correcta administración de los procesos y recursos, así como de la generación de la información necesaria para la misma.

\section{Elementos del costo.}

Los elementos del costo para el Salón los Maderos se identifican entre directos e indirectos.

En la tabla 1, se determinan los costos directos e indirectos para proceder a la distribución por actividades de los CIF. Como se observa en la tabla 1 , el costo unitario es de $\$ 15,41$ determinado por el costo tradicional, sin embargo, bajo este método no se puede identificar en qué departamento esta mayormente la carga. 
Tabla 1. Costos directos e indirectos Salón los Maderos.

\begin{tabular}{lr|lr}
\hline Costos directos & Costo anual & Costos indirectos & \multicolumn{1}{c}{$\begin{array}{c}\text { Costo } \\
\text { anual }\end{array}$} \\
\hline $\begin{array}{l}\text { Materia prima } \\
\text { - ingredientes chancho }\end{array}$ & $34.200,00$ & - mesero & \\
Barbosa & & - facturadora, cobrador & $7.000,00$ \\
& & - ayudante limpieza & $6.000,00$ \\
Mano de obra & $12.000,00$ & & \\
- chef & $7.500,00$ & CIF & \\
- asistente de cocina & & - gas industrial & \\
& & - mantenimiento & \\
& & - insumos varios & 800,00 \\
& & Total & 300,00 \\
\hline Total & $\mathbf{5 3 . 7 0 0 , 0 0}$ & $\mathbf{2 1 . 8 0 0 , 0 0}$ \\
\hline Unidades producidas & 4.900 & Unitario & 15,41
\end{tabular}

\section{Identificación de actividades, centros de costos e inductores.}

Una vez identificadas las actividades estas se relacionan con los centros de costos para determinar los inductores de recursos, y asignar los costos indirectos por actividad.

Tabla 2. Centros de costos y actividades Salón los Maderos

\begin{tabular}{llll}
\hline \multicolumn{1}{c}{ Centro de costos } & \multicolumn{1}{c}{ Actividades } & \multicolumn{1}{c}{ Inductores } & \multicolumn{1}{c}{ Responsables } \\
\hline Preparación & lavar & horas hombre & ayudante de cocina \\
& cortar & horas hombre & ayudante de cocina \\
& pesar & horas hombre & ayudante de cocina \\
& condimentar & horas hombre & chef \\
Cocción & cocinar & horas hombre & chef \\
Emplatado & decorar & horas hombre & chef \\
& servir & horas hombre & chef \\
Servicio al cliente y logística & pedido del cliente & número de pedidos & mesero \\
& orden a cocina & número de órdenes & mesero \\
& servicio a la mesa & horas hombre & mesero \\
& facturación & numero de facturas & facturador \\
& limpieza & horas hombre & ayudante de limpieza \\
\hline
\end{tabular}


Tabla 3. Distribución del CIF a las actividades.

\begin{tabular}{|c|c|c|c|c|c|c|c|c|c|}
\hline Costos indirectos & & to anual & Inductores & Unidad & Pedido & servicio & facturación & limpieza & cocinar \\
\hline Mesero & $\$$ & $6.000,00$ & horas hombre & $\$ 3,13$ & $\$ 3.000,00$ & $\$ 3.000,00$ & & & \\
\hline Facturador, cobrador & $\$$ & $7.500,00$ & número de facturas & $\$ 1,53$ & & & $\$ 7.500,00$ & & \\
\hline Ayudante de limpieza & $\$$ & $6.000,00$ & horas hombre & $\$ 1,22$ & & & & $\$ 6.000,00$ & \\
\hline Gas industrial & $\$$ & $1.200,00$ & número de órdenes & $\$ 0,24$ & & & & & $\$ 1.200,00$ \\
\hline Mantenimiento & $\$$ & 800,00 & horas hombre & $\$ 0,16$ & & & & 800,00 & \\
\hline Insumos varios & $\$$ & 300,00 & horas hombre & $\$ 0,06$ & & & & 300,00 & \\
\hline Total & $\$$ & $21.800,00$ & & & $\$ 3.000,00$ & $\$ 3.000,00$ & $\$ 7.500,00$ & $\$ 7.100,00$ & $\$ 1.200,00$ \\
\hline Unidades producidas & & 4900 & & & & & & & \\
\hline
\end{tabular}

Los CIF son asignados por medio de los inductores de costos a cada actividad.

Tabla 4. Determinación del costo total por centros de costos.

\begin{tabular}{|c|c|c|c|c|}
\hline Centro de costos & Actividades & Costos directos & costos indirectos & costo total \\
\hline \multirow[t]{4}{*}{ Preparación } & Lavar & $4.000,00$ & & 4000 \\
\hline & Cortar & $2.500,00$ & & 2500 \\
\hline & Pesar & $1.000,00$ & & 1000 \\
\hline & Condimentar & $1.000,00$ & & 1000 \\
\hline Cocción & Cocinar & $42.200,00$ & 1200 & $43.400,00$ \\
\hline \multirow[t]{2}{*}{ Emplatado } & Decorar & $2.000,00$ & & $2.000,00$ \\
\hline & Servir & $1.000,00$ & & $1.000,00$ \\
\hline \multirow[t]{6}{*}{$\begin{array}{l}\text { Servicio al cliente y } \\
\text { logística }\end{array}$} & Pedido del cliente & & 3000 & $3.000,00$ \\
\hline & Servicio a la mesa & & 3000 & $3.000,00$ \\
\hline & Facturación & & 7500 & $7.500,00$ \\
\hline & Limpieza & & 7100 & $7.100,00$ \\
\hline & Total & $53.700,00$ & 21800 & $75.500,00$ \\
\hline & Unidades: 4900 & 10,96 & 4,45 & 15,41 \\
\hline
\end{tabular}

Como se observa comparando la tabla 1 con la tabla 4 el costo unitario y el coste total no cambia, se mantiene. El método de acumulación no cambia el costeo, lo que hace es determinar de manera estratégica que actividades son las que consumen más recursos.

\section{Conclusiones}

- La gestión de costos $\mathrm{ABC}$ tiene su evolución, y entre los sistemas y métodos de costeo, se establece el sistema de gestión de costos $\mathrm{ABC} / \mathrm{ABM}$ que no solo costea la producción o los servicios; sino que evalúa los procesos, recursos y actividades que intervienen en la producción o prestación de un servicio, con el objetivo de competir en cuanto al servicio 
que reciben los clientes y a la eliminación de gastos o costos innecesarios, elevando de esta manera las utilidades de los inversionistas en las empresas.

- La identificación de las actividades de los principales procesos constituye el primer paso para una correcta gestión de costos $\mathrm{ABC} / \mathrm{ABM}$, puesto que solo identificando claramente las operaciones que agregan valor y cuales deben ser suprimidas, se pueden realizar reestructuraciones hacia un costeo más completo y fiable, asi como establecer las estrategias para la optimizacion de recursos.

- El diagnóstico realizado en el Salón los Maderos deja en evidencia la deficiente gestión de talento humano que se practica, debido a que, no cuenta con procesos adecuados de contratación ni capacitación, que aseguran contar con personal altamente competente . Otro aspecto significativo que se identificó fue la determinación empírica de los costos de producción y la carencia de información financiera que aporte a la toma de decisiones a nivel estratégico, táctico y operativo.

- La implementación de un modelo de gestion de costos basado en actividades permitirá que en el restaurante Los Maderos se determine el valor que representa cada actividad que se ejecuta sin dejar de lado la cuantificación de todos los rubros que intervienen en la elaboración de los producto y servicios que oferta, paralelo a esto, con la identificación de gastos, actividades y procesos innecesarios, la gerencia podrá tomar decisiones acertadas.

\section{Referencias Bibliográficas}

Servicio de Rentas Internas. (2015). Plan Estrategico Institucional 2016-2019. Quito: Servicio de Rentas Internas.

Acevedo, S. (1998). Planificación Tributaria y Administración de Capital de Trabajo. Colombia: Norma.

Adame Welsh, R. A. (2000). Costeo basado en actividades (ABC): conceptos teóricos y metodología de implementación, Tesis de maestría. Monterrey.

Altajona Quijano, T. (2009). Libro práctico sobre la contabilidad de costos. Bucaramanga: UDI PORTER.

Amorós, N. (1965). La elusión y la evasión tributaria. Madrid: Revista de Derecho Financiero y Hacienda Pública. 
Bendersky, E. (2002). ABC-ABM gestión de costos por actividades. Buenos Aires: Editorial de las Ciencias.

Bernal, C. (2010). Metodología de la investigación. Bogotá: Pearson.

Blocher, E., Stout, D., Cokins, G., \& Chen, K. (2008). Administración de Costos. México: McGraw-Hill.

Bravo Valdivieso, M., \& Ubidia Tapia, C. (2013). Contabilidad de costos. Quito: Editora "Escobar Impresores".

Calleja, F. (2013). Costos. Naucalpan de Juárez: Pearson.

Cámara, L. (2005). Planeación Estrategica. Madrid: CIDEAL.

Cazau, P. (2006). Introducción a la Investigación en Ciencias Sociales 3ra Edición. Buenos Aires.

Cooper, R., \& Kaplan, R. (1991). The design of cost management systems. New Jersey: Practice-Hall.

Crespo, A. (2016). La Presión Fiscal en el Ecuador: Análisis de la Carga Tributaria (Tesis Maestría). Guayaquil: Instituto de Altos Estudios Nacionales.

Cuervo Tafur, J., Osorio Agudelo, A., \& Duque, M. (2013). Costeo basado en actividades ABC Gestión basada en actividades ABM. Bogotá: ECOE.

Cuevas, F. (2001). Contabilidad de costos enfoque gerencial y de gestión. Bogotá: Quebecor Word Bogotá S.A.

Cusgüen, E. (1998). Planificación del tributo, impuestos. Madrid: Colección Europa.

Dávila, G. (2006). El razonamiento inductivo y deductivo dentro del proceso investigativo en ciencias experimentales y sociales. Laurus, 184-186. Recuperado el 10 de 11 de 2018, de <http://www.redalyc.org/articulo.oa?id=76109911>

Delgado, G. (2010). Conceptos y metodología de la investigación histórica. Revista Cubana de Salud Pública, 9-18.

Delgado, G. (2010). Conceptos y metodología de la investigación histórica. Revista Cubana de Salud Pública, 36(1), 9-18. Recuperado el 12 de noviembre de 2018, de http://scielo.sld.cu/scielo.php?script=sci_arttext\&pid=S0864$34662010000100003 \& \operatorname{lng}=$ es\&tlng=pt.

Diesing, P. (1972). Patterns of Discovery in the Social Sciences. Londres: Rotledge \& Kegan Paul.

Dirección Nacional de Investigación y Estudios. (2016). Breve análisis societario del sector de la construcción del Ecuador 2014-2015. Quito: Superintendencia de Compañías, valores y seguros.

Drucker, P. (2006). La toma de decisiones. Barcelona: Ediciones Deusto.

Dupont. (17 de 03 de 2018). Dupont.com. Obtenido de Dupont.com: http://www2. dupont.com/Phoenix_Heritage/en_US/1918_detail.html 
Emery, D., Finnerty, J., \& Stowe, J. (2000). Fundamentos de la administración financiera. Naucalpan de Juárez, Edo. de México: Prentice hall hispanoamericana S.A.

Escudero, A. (2009). La revolución industrial: Una nueva era. Madrid: ANAYA.

Fierro, A. (2007). Diagnóstico empresarial. Bogotá: ECOE ediciones.

Foundation, I. (2016). NIC 38.

Galárraga, A. (2002). Fundamentos de la Planificación Tributaria. Carácas: Editorial Jurídica Venezolana.

García Colin, J. (2008). Contabilidad de Costos. México: McGraw-Hill Interamericana.

García, O. (2014). Fórmula Du Pont y su rentabilidad, vista desde la óptica administrativa. Inquietud Empresarial, 89-113.

Gareth, J., \& George, J. (2010). Administración contemporánea. México D.F: McGrawHill.

Goldratt, E. (2008). Necesario pero no suficiente. México: Granica.

Gómez Bravo, O., \& Zapata Sánchez, P. (1998). Contabilidad de costos. Bogotá: Impreandes Presencia S.A.

Guizar, R. (2013). Desarrolo organizacional, principios y aplicaciones. México D.F.: Mc Graw Hill.

H. Congreso Nacional, E. (30 de 11 de 2007). LORTI. LORTI. Quito, Pichincha, Ecuador: RO. 223.

Hansen, D., \& Mowen, M. (2007). Administración de costos: contabilidad y control. México D.F: Cengage learning editores S.A. Obtenido de http://biblioteca.soymercadologo.com/wpcontent/uploads/2016/08/Administraci\%C3\%B3n-de-costos-5ed-Don-R.-Hansen-y-MaryanneM.-Mowen.pdf

Herbert, S. (1977). La nueva ciencia de la decisión gerncial. Buenos Aires: El Ateneo.

Hernández, R., Fernández, C., \& Baptista, P. (2008). Metodología de la Ivestigación. Mexico: McGrawHill.

Hidalgo, A. (20 de 12 de 2009). https://www.urbe.edu. Obtenido de Aspectos generales de la planificación tributaria en Venezuela: https://www.urbe.edu/UDWLibrary/ArticulosAdvance.do?operator=EMPTY\&tag=100\&word=H idalgo\%20de\%20Camba,\%20Alejandra

Horngren, C., Datar, S., \& Rajan, M. (2012). Contabilidad de costos: un enfoque gerencial. México: Pearson.

IFRS. (2016). NIC 36.

Ilescas, C. (2012). Activos no corrientes. Loja: EDILOJA Cia. Ltda. 
Illescas, C. (2012). Activos no corrientes. Loja: EDILOJA Cia. Ltda.

Instituto de Desarrollo Industrial, Tecnológico y de Servicio. (2009). Informe Final: Sector Construcción, Parte. Argentina: inet.

Instituto Nacional de Estadistica y Censos INEC. (10 de Diciembre de 2012). La Industria de la Construcción es el mayor empleador del mundo. Obtenido de ecuadorencifras.gob.ec: http://www.ecuadorencifras.gob.ec/wp-content/descargas/Infoconomia/info10.pdf

Instituto nacional de estadísticas y censos. (2015). INEC. Recuperado el 14 de 04 de 2019, de http://www.ecuadorencifras.gob.ec/documentos/webinec/Estadisticas_Economicas/Encuesta_Servicios/Servicios_2015/2015_ES_Resumen_Ejecutivo .pdf

Kaplan, R., \& Johnson, T. (1988). La contabilidad de Costes: Auge y Caída de la Contabilidad de Gestión. Barcelona: Plaza y Jánes Editores.

Laporta Pomi, R. (2016). Costos y Gestión Empresarial. Incluye costos con ERP. Bogotá: ECOE Ediciones.

Lawrence, J., \& Chad, J. (2012). Principios de administración financiera. México: Pearson.

Llanos, N. (05 de 11 de 2018). ACADEMIA. Obtenido de ACADEMIA.EDU: http://www.academia.edu/5075869/CLASES_Y_TIPOS_DE_INVESTIGACION_Y_SUS_CAR ACTERISTICAS

López, F. (2003). Decisiones empresariales y sentido común. Madrid: McGraw-Hill.

López, K. (2017). La planificación tributaria como herramienta financiera para la toma de decisiones en el sector inmobiliarioa. Quito.

Mathews, M. R., \& Perera, M. H. (1991). Accounting theory and development. Hong Kong: Chapman \& Hall.

Medizabal, L. (2014). Análisis Jurídico del la Evación y Elución Fiscal en Guatemala (Tesis de Posgrado). Guatemala: Universidad Rafael Landívar.

Ministerio de Justicia, Derechos Humanos y Cultos. (02 de 10 de 2014). Código Orgánico Integral Penal. Código Orgánico Integral Penal. Quito, Pichincha, Ecuador: RO 180.

Moody, P. (1983). Decision making: methods for better decisions. New York: Mc. Graw. Hill.

Mora, F. (04 de 2013). Análisis estratégico de la segmentación de los restaurantes de primera clase, de la ciudad de Cuenca antes y después de la aplicación de los descriptores de segmentación. Obtenido de http://dspace.uazuay.edu.ec/bitstream/datos/3447/1/10146.PDF

Moriarity, S., \& Allen, C. P. (1990). Contabilidad de Costos. México D.F: Compañia editorial Continental, S.A DE C.V Mexico. 
Orama, A., Pérez, O., \& Quesada, M. (2010). Etapas en la determinación del coste por actividades: caso Hotel Meliá Varadero. Papers de turisme, 41-55. Obtenido de http://www.papersdeturisme.gva.es/ojs/index.php/Papers/article/viewFile/82/76

Osar, C., \& Ramos, N. (20 de 12 de 2017). El portal fiscal y el control tributario. Obtenido de El portal fiscal y el control tributario: www.nerlassociados.com

Otros, \& Älvarez López, J. (1996). Contabilidad de Gestión Avanzada. Madrid: McGraw-Hill.

Padilla Alvarez, G. e. (2003). Tutorial para la Asignatura de Costos y Presupuestos. México D.F: Fondo Editorial FCA.

Parra, A. (2007). Planeación Tributaria y Organización Empresarial. Colombia: LEGIS.

Perdomo, A. (1993). Toma de decisiones financieras: contabilidad de decisiones. México D.F: Ecasa.

Pérez Barral, O. (2009). Origen del sistema de gestión y costos basado en actividades (ABC/ABM). Revista Avanzada Científica, 1-21.

Pérez, A. (2015). Dieño e implantación de un modelo ABC/ABM para una empresa hotelera. Cálculo de costes e indicadores de gestión (tesis doctoral). Málaga: Publicaciones y Divulgación Científica. Universidad de Málaga.

Polimeni, R. S., Fabozzi, F. J., \& Adelberg, A. H. (1997). Contabilidad de costos. Conceptos y aplicaciones para la toma de decisiones gerenciales. Colombia: McGraw-Hill.

Quintero Ortiz, B. C. (2013). Costeo para un restaurante gourmet en Colombia. Colombia.

Rabadán, A., Cid, A., \& Leguey, S. (2013). Métodos de decisión empresarial. Madrid: Delta.

Ramirez Padilla, D. (2008). Contabilidad administrativa. Mexico, D.F.

Ramirez, A. (2003). Metodología de la investigación científica. Bogotá: Pontificia Universidad Javeriana.

Reyes Perez, E. (2002). Contabilidad de costos. México: Editorial Limusa.

Rivas, N., \& Vergara, S. (2002). Planificación Tributaria. Santiago de Chile: Magril.

Robbin, S. (1987). Administración teórica y práctica. México: Prentice-Hall Hispanoamérica S.A.

Ruiz, J. (1998). El Fraude de Ley y Otros Supuestos de Elusión Fiscal. Valencia: CISS S.A.

Sánchez, L., \& Hablich, F. (2018). Contribución de la planificación. Espacios, 15.

Sarmiento, R. (2005). Contabilidad de costos. Quito: Editorial Voluntad.

Sautu, R., Boniolo, P., Dalle, P., \& Elbert, R. (2005). Manual de metodología: construcción del marco teórico, formulación de los objetivos y elección de la metodología. Buenos Aires: Clacso libros. 
Secretaría Nacional de Planificación y Desarrollo. (22 de 09 de 2017). Obtenido de http://www.planificacion.gob.ec/wp-content/uploads/downloads/2017/10/PNBV-26-OCTFINAL_0K.compressed1.pdf

Soto, E., \& Cárdenas, J. (2007). Ética en las orgqanizaciones. México D.F: McGraw-Hill.

SRI. (15 de 03 de 2018). Visores Tributarios. Obtenido de Visores Tributarios: http://visoresestadisticos.sri.gob.ec/qap/single/?appid=73ae68ff-7352-40cb-8b1a053e4ec0838a\&sheet=6ac5c195-1f61-477a-8f4f-bb6801e4b73f\&lang=es-ES\&opt=currsel

Stout, D. E., Cokings, G., Chen, K., \& Blocher, E. (2008). Administración de costos. México: McGrawHill Interamericana.

Taylor, F. (1969). Principios de la administración científica. México: Herrero Hnos S.A.

Tipker, K. (2002). Moral Tributaria del Estado y de los contribuyentes. Madrid: Marcial Pons.

Toro López, F. J. (2010). Costos ABC y presupuestos: Herramientas para la productividad. Bogotá: ECOE Ediciones.

Torres Salinas, A. (1996). Contabilidad de costos: análisis para la toma de decisiones. México: McGraw-Hill.

Uribe, R. (2011). Costos para la toma de decisiones. Bogotá: McGraw-Hill Interamericana.

Van, J. (1997). Administración Financiera. Naucalpan de Juárez, Edo. de México: Prentice hall hispanoamericana S.A.

Vanderberck, E., \& Mitchell, M. (2016). Principios de contabilidad de costos. México: Cengage Learning.

Villafuerte, D. (2006). Manual Metodologico para el Investigador Cientifico. Parú: U.N.S.A.

Weiss, W. (1987). Guía práctica para la toma de decisiones. Colombia: Norma S.A.

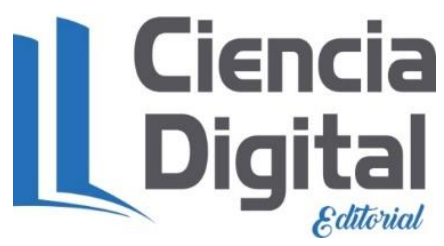




\section{PARA CITAR EL ARTÍCULO INDEXADO.}

Guerrero Romero, J., Narváez Zurita, C., Ormaza Andrade, J., \& Erazo Álvarez, J. (2019). El costeo ABC como estrategia de gestión empresarial en el sector de servicios. Caso: Salón los Maderos. Visionario Digital, 3(2.1.), 215-235.

https://doi.org/10.33262/visionariodigital.v3i2.1.553

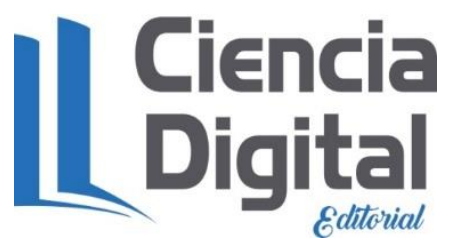

El artículo que se publica es de exclusiva responsabilidad de los autores y no necesariamente reflejan el pensamiento de la Revista Ciencia Digital.

El artículo queda en propiedad de la revista y, por tanto, su publicación parcial y/o total en otro medio tiene que ser autorizado por el director de la Revista Ciencia Digital.
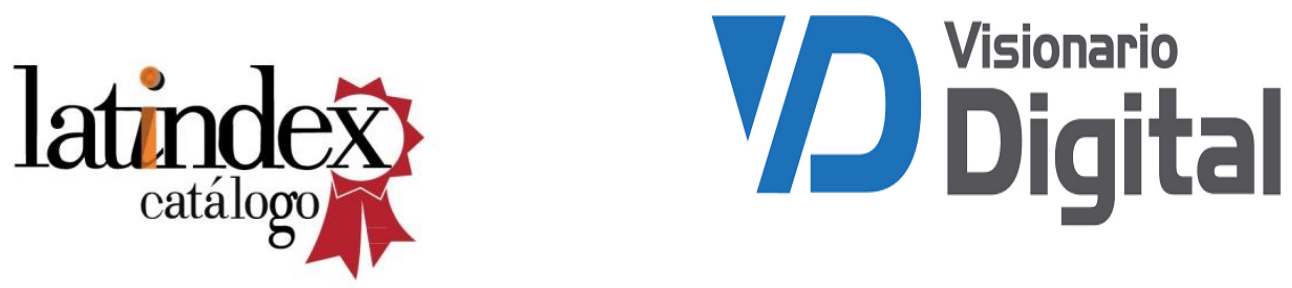